\title{
GUIDANCE AND NAVIGATION FOR RENDEZVOUS WITH AN UNCOOPERATIVE TARGET
}

\section{J. Telaar, C. Schlaile, and J. Sommer}

\author{
Airbus Defence and Space \\ 1 Airbus-Allee, Bremen 28199, Germany
}

\begin{abstract}
This paper presents a guidance strategy for a rendezvous with an uncooperative target. In the applied design reference mission, a spiral approach is commanded ensuring a collision-free relative orbit due to $e / i$-vector separation. The dimensions of the relative orbit are successively reduced by $\Delta v$ commands which at the same time improve the observability of the relative state. The navigation is based on line-of-sight measurements. The relative state is estimated by an extended Kalman filter (EKF). The performance of this guidance and navigation strategy is demonstrated by extensive Monte Carlo simulations taking into account all major uncertainties like measurement errors, $\Delta v$ execution errors, and differential drag.
\end{abstract}

\section{INTRODUCTION}

The rendezvous with an uncooperative target is a major challenge for space debris removal missions, for Mars and Lunar sample return missions, and similar missions. The focus of this paper is on the transition from ground based absolute navigation to autonomous relative navigation. The knowledge of the relative state is mandatory to determine the $\Delta v$ commands to approach the uncooperative target on a safe, collision-free trajectory. As long as the distance between chaser and target is larger than the operational range of the chaser's relative navigation sensors, the target's orbital parameters or position and velocity are determined from ground, e.g., by ranging, Doppler radar, and/or differential Doppler radar. As soon as the distance becomes small enough and the target has been detected by the chaser's relative navigation sensors, the target tracking is started and the relative state can be estimated on the basis of these measurements. If a camera is used, only line-of-sight measurements are available. The

(C) The Authors, published by EDP Sciences. This is an open access article distributed under the terms of the Creative Commons Attribution License 4.0 (http://creativecommons.org/licenses/by/4.0/). 
lack of range measurements leads to the problem that the relative state is not fully observable as long as no manoeuvres are performed by the chaser. Fortunately, the chaser has to perform manoeuvres anyway to approach to the target. However, some strategies require much propellant, others are not passively safe. The presented guidance and navigation strategy is close to the optimum in terms of propellant consumption and the resulting trajectory is always passively safe.

The guidance strategy is based on relative orbital elements (ROE) with $e / i$ vector separation. The ROE are a solution of the Clohessy-Wilthire equations [1]. The concept of $e / i$-vector separation had originally been designed for the safe collocation of geostationary satellites, but has recently been applied also for proximity operations of Low Earth Orbit (LEO) satellites (e.g., Tandem-X [2] and PRISMA [3]).

The EKF is sufficient for the estimation of ROE. Filters like the unscented Kalman filter require more computational effort without providing significantly better results for this application $[4,5]$.

\section{RELATIVE ORBITAL ELEMENTS}

The relative position and velocity of two satellites orbiting the Earth can be described by ROE. Details on the derivation of the ROE can be found in [6]. The ROE are more intuitive than relative position and velocity in Cartesian coordinates as they describe the geometry of the motion of the chaser around the target. The ROE are stable whereas the relative position and velocity are fluctuating with the orbital motion. Therefore, the ROE are more suitable for guidance and control. They are defined as

$$
\delta \vec{a}=\left(\begin{array}{c}
\delta a \\
\delta \lambda \\
\delta e_{x} \\
\delta e_{y} \\
\delta i_{x} \\
\delta i_{y}
\end{array}\right)=\left(\begin{array}{c}
\frac{\Delta a}{a} \\
\Delta u+\Delta \Omega \cos (i) \\
\Delta e_{x} \\
\Delta e_{y} \\
\Delta i \\
\Delta \Omega \sin (i)
\end{array}\right)
$$

where $a$ denotes the semimajor axis; $u$ is the argument of latitude being the sum of argument of perigee and mean anomaly; $\Omega$ is the right ascension of the ascending node; $i$ is the inclination; $\Delta$ is the difference of the particular quantity between chaser and target; and $\delta \lambda$ denotes the relative mean longitude.

The eccentricity vector components $e_{x}$ and $e_{y}$ are defined as $e \cos \omega$ and $e \sin \omega$ where $e$ is the eccentricity and $\omega$ is the argument of perigee. 
The ROE can be transformed in radial, tangential, and normal (RTN) components of relative position and velocity [6]:

$$
\left(\begin{array}{c}
r_{R} \\
r_{T} \\
r_{N} \\
v_{R} \\
v_{T} \\
v_{N}
\end{array}\right)=\left(\begin{array}{c}
a \delta a-a \delta e_{x} \cos u-a \delta e_{y} \sin u \\
a \delta \lambda-\frac{3}{2}\left(u-u_{0}\right) a \delta a+2 a \delta e_{x} \sin u-2 a \delta e_{y} \cos u \\
a \delta i_{x} \sin u-a \delta i_{y} \cos u \\
n_{\omega} a \delta e_{x} \sin u-n_{\omega} a \delta e_{y} \cos u \\
-\frac{3}{2} n_{\omega} a \delta a+2 n_{\omega} a \delta e_{x} \cos u+2 n_{\omega} a \delta e_{y} \sin u \\
n_{\omega} a \delta i_{x} \cos u+n_{\omega} a \delta i_{y} \sin u
\end{array}\right) .
$$

Here, $n_{\omega}$ denotes the mean angular rate of the orbital motion:

$$
n_{\omega}=\sqrt{\frac{\mu_{E}}{a^{3}}}
$$

where $\mu_{E}$ is the gravitational parameter of the Earth.

The basic idea behind the concept of $e / i$-vector separation may be explained as follows. Assume two identical orbits with inclination larger than zero. Then, pick one and modify the eccentricity while keeping the semimajor axis constant. Now, the two orbits have two points of intersection. Shift the argument of perigee such that the apogee and perigee are at the maximal and minimal latitude or vice versa. The two points of intersection of the two orbits are now at the equator. Now, modify the right ascension of the ascending node. Then, the two orbits have no points of intersection anymore. The resulting relative motion corresponds to the chaser's flight around the target.

The $e / i$-vector separation is especially suited for the far range formation flight as it generates a passively safe formation by introducing a relative eccentricity and a relative inclination vector such that chaser and target are separated in cross-track and radial directions. The concept is driven by the fact that, in general, the uncertainty in predicting the along-track separation is much higher than in predicting the other two components. Thus, both spacecraft, chaser and target, are separated in radial and cross-track directions in order to avoid a collision. Maximal safety is achieved if the vectors $\delta e$ and $\delta i$ are parallel or antiparallel. The concept of $e / i$-vector separation is shown in Fig. 1 .

The major disturbances are due to differential drag and $J_{2}$-term representing the gravitational effects due to the flattening of the Earth. The differential drag plays a major role in very low Earth orbits, i.e., below $500 \mathrm{~m}$, if the ballistic coefficients of chaser and target are different; otherwise, it might be neglected. The influence of the $J_{2}$-term can be minimized by proper arrangement of the relative orbit, i. e., $a \delta i_{x}=0$. 

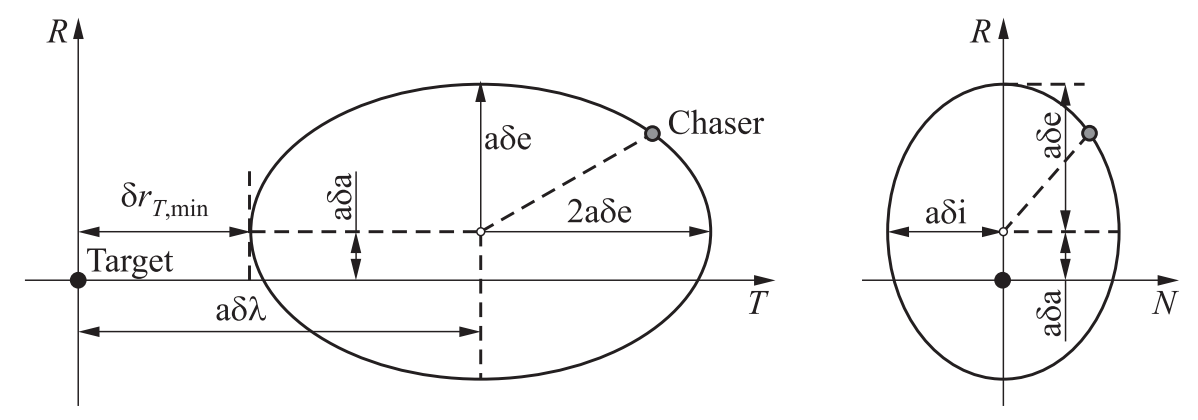

Figure 1 Relative orbital elements and $e / i$-vector separation

\section{GUIDANCE STRATEGY}

The rendezvous with an uncooperative target can be subdivided into several mission segments. The phasing is based on absolute navigation. During this phase, the chaser's orbit is determined using the chaser's global positioning system (GPS) data and the target's orbit by Two-Line Element (TLE) data. The relative orbit and the manoeuvres required for the phasing are calculated with these data. The phasing ends in the Far Formation Flying (FFF) with a switch on of the far range camera. After confirmation that the target is correctly identified, the approach from FFF to the Initial Aim Point (IAP) begins. The primary relative navigation sensor used during the approach is the far range camera which provides line-of-sight measurements. At the IAP, the Light Detection and Ranging (LIDAR) sensor is switched on which provides line-of-sight and range measurements. The rationale for the sensor selection is its maximal range. The far range camera allows measurements at approximately 5-kilometer distance or more, depending on the size of the target and the selected camera, whereas the LIDAR is limited to approximately 1-kilometer distance, which is below the TLE accuracy for the along-track position.

The goal of the guidance strategy presented in this paper is the approach from FFF to IAP in a safe, collision-free manner, while the propellant consumption is minimized. Additionally, the relative navigation filter requires some manoeuvres to ensure observability of the relative state. The guidance strategy foresees a spiral approach. In the first along-track manoeuvre, a drift towards the target is established. This manoeuvre also reduces the relative orbit in radial direction. Afterwards, the relative orbit is reduced by radial and cross-track manoeuvres while the drift is kept constant. A final along-track manoeuvre terminates the drift and reduces the radial component of the relative orbit to the desired final value. The repeated radial and cross-track manoeuvres enhance the observability of the relative state and have the advantage that the 
relative orbit becomes smaller as the navigation solution becomes better with time.

\subsection{Guidance Algorithm}

The effect of impulsive manoeuvres on the ROE for circular orbits is:

$$
a \delta \vec{\alpha}(u)=\left(\begin{array}{c}
a \delta a_{0} \\
a \delta \lambda_{0}-\frac{3}{2}\left(u-u_{0}\right) a \delta a_{0} \\
a \delta e_{x 0} \\
a \delta e_{y 0} \\
a \delta i_{x 0} \\
a \delta i_{y 0}
\end{array}\right)+\frac{1}{n_{\omega}}\left(\begin{array}{c}
2 \delta v_{T} \\
-2 \delta v_{R}-3\left(u-u_{T}\right) \delta v_{T} \\
\delta v_{R} \sin u_{R}+2 \delta v_{T} \cos u_{T} \\
-\delta v_{R} \cos u_{R}+2 \delta v_{T} \sin u_{T} \\
\delta v_{N} \cos u_{N} \\
\delta v_{N} \sin u_{N}
\end{array}\right)
$$

Furthermore, it is possible to distribute radial and cross-track manoeuvres to several orbits without changing the total $\delta v$ or the final relative orbit. The transition from the initial relative orbit $a \delta \vec{\alpha}_{0}$ to the final relative orbit $a \delta \vec{\alpha}_{F}$ can be achieved by any solution of the form:

$$
\begin{aligned}
a \delta \delta \vec{\alpha}=a \delta \vec{\alpha}_{F}-a \delta \vec{a}_{0} \\
=\frac{1}{n_{\omega}}\left(\begin{array}{c}
3 \sum \delta v_{T} \\
-\frac{3}{2}\left(u_{F}-u_{0}\right) n_{\omega} a \delta a_{0}-2 \sum \delta v_{R}-2 \sum\left(u_{F}-u_{T}\right) \delta v_{T} \\
\sum \delta v_{R} \sin u_{R}+2 \sum \delta v_{T} \cos u_{T} \\
-\sum \delta v_{R} \cos u_{R}+2 \sum \delta v_{T} \sin u_{T} \\
\sum \delta v_{N} \cos u_{N} \\
\sum \delta v_{N} \sin u_{N}
\end{array}\right)
\end{aligned}
$$

where $u_{F}$ is the final argument of latitude which is set to the last manoeuvre. Note that the cross-track component in Eq. (2) is decoupled from the first four ROE. The argument of latitude $u_{N}$ and the $\delta v_{N}$ for cross-track manoeuvres are calculated by:

$$
\begin{aligned}
\tan u_{N} & =\frac{a \delta \delta i_{y}}{a \delta \delta i_{x}} \\
\delta v_{N} & =\frac{1}{n_{\omega}} \sqrt{a \delta \delta i_{x}^{2}+a \delta \delta i_{y}^{2}}
\end{aligned}
$$

where $\delta$ denotes the difference between the final and the initial quantity; accordingly, $\delta \delta$ denotes the difference between final and initial ROE. The $\delta v_{N}$ can be split to manoeuvres every half orbital period. Every second manoeuvre is in 
the opposite direction of the first manoeuvre. The changes in relative semimajor axis $a \delta \delta a$, the relative along-track position $a \delta \delta \lambda$, and the components $a \delta \delta e_{x}$ and $a \delta \delta e_{y}$ are coupled and can be written in the following form:

$$
\left(\begin{array}{c}
a \delta \delta a \\
a \delta \delta \lambda+\frac{3}{2}\left(u_{F}-u_{0}\right) a \delta a_{0} \\
a \delta \delta e_{x} \\
a \delta \delta e_{y}
\end{array}\right)=\frac{1}{n_{\omega}}\left(\begin{array}{c}
2 \sum \delta v_{T} \\
-2 \sum \delta v_{R}-3 \sum\left(u_{F}-u_{T}\right) \delta v_{T} \\
\sum \delta v_{R} \sin u_{R}+2 \sum \delta v_{T} \cos u_{T} \\
-\sum \delta v_{R} \cos u_{R}+2 \sum \delta v_{T} \sin u_{T}
\end{array}\right)
$$

The arguments of latitude of the radial and tangential manoeuvres are calculated by:

$$
\begin{aligned}
\tan u_{R} & =\frac{a \delta \delta e_{x}}{-a \delta \delta e_{y}} \\
\tan u_{T} & =\frac{a \delta \delta e_{y}}{a \delta \delta e_{x}} .
\end{aligned}
$$

The manoeuvres may be shifted by multiples of $\pi$. Equation (3) can be rewritten as

$$
A \vec{x}=\vec{b}
$$

where the matrix $A$ is composed of one column per radial and one column per along-track manoeuvre:

$$
A=\frac{1}{n_{\omega}}\left[\begin{array}{cccccc}
0 & \cdots & 0 & 2 & \cdots & 2 \\
-2 & \cdots & -2 & 3\left(u_{F}-u_{T 1}\right) & \cdots & 3\left(u_{F}-u_{T n_{T}}\right) \\
\sin u_{R 1} & \cdots & \sin u_{R n_{R}} & 2 \cos u_{T 1} & \cdots & 2 \cos u_{T n_{T}} \\
-\cos u_{R 1} & \cdots & -\cos u_{R n_{R}} & 2 \sin u_{T 1} & \cdots & 2 \sin u_{T n_{T}}
\end{array}\right]
$$

$\vec{x}$ contains the vector of radial and tangential manoeuvres:

$$
\vec{x}=\left(\begin{array}{c}
\delta v_{R 1} \\
\vdots \\
\delta v_{R n_{R}} \\
\delta v_{T 1} \\
\vdots \\
\delta v_{T n_{T}}
\end{array}\right) ;
$$

and $\vec{b}$ contains the desired changes of the ROE:

$$
\vec{b}=\left(\begin{array}{c}
a \delta \delta a \\
a \delta \delta \lambda+\frac{3}{2}\left(u_{F}-u_{0}\right) a \delta a_{0} \\
a \delta \delta e_{x} \\
a \delta \delta e_{y}
\end{array}\right)
$$


Then, the solution of Eq. (4) is calculated by:

$$
\vec{x}=\left(A^{\mathrm{T}} A\right)^{-1} A^{\mathrm{T}} \vec{b} .
$$

The $\delta v$ commands are generated by Eq. (5). During the approach, these commands have to be updated due to navigation and control errors. The commands for the upcoming orbital period are executed. Then, the $\delta v$ commands are updated.

\subsection{Optimal Propellant Consumption Strategy}

The propellant consumption is strongly dependent on the guidance strategy.

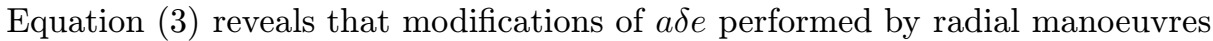
cost twice as much $\delta v$ as if it is performed by along-track manoeuvres. The difference is even larger if radial manoeuvres are used for large changes in $a \delta \lambda$.
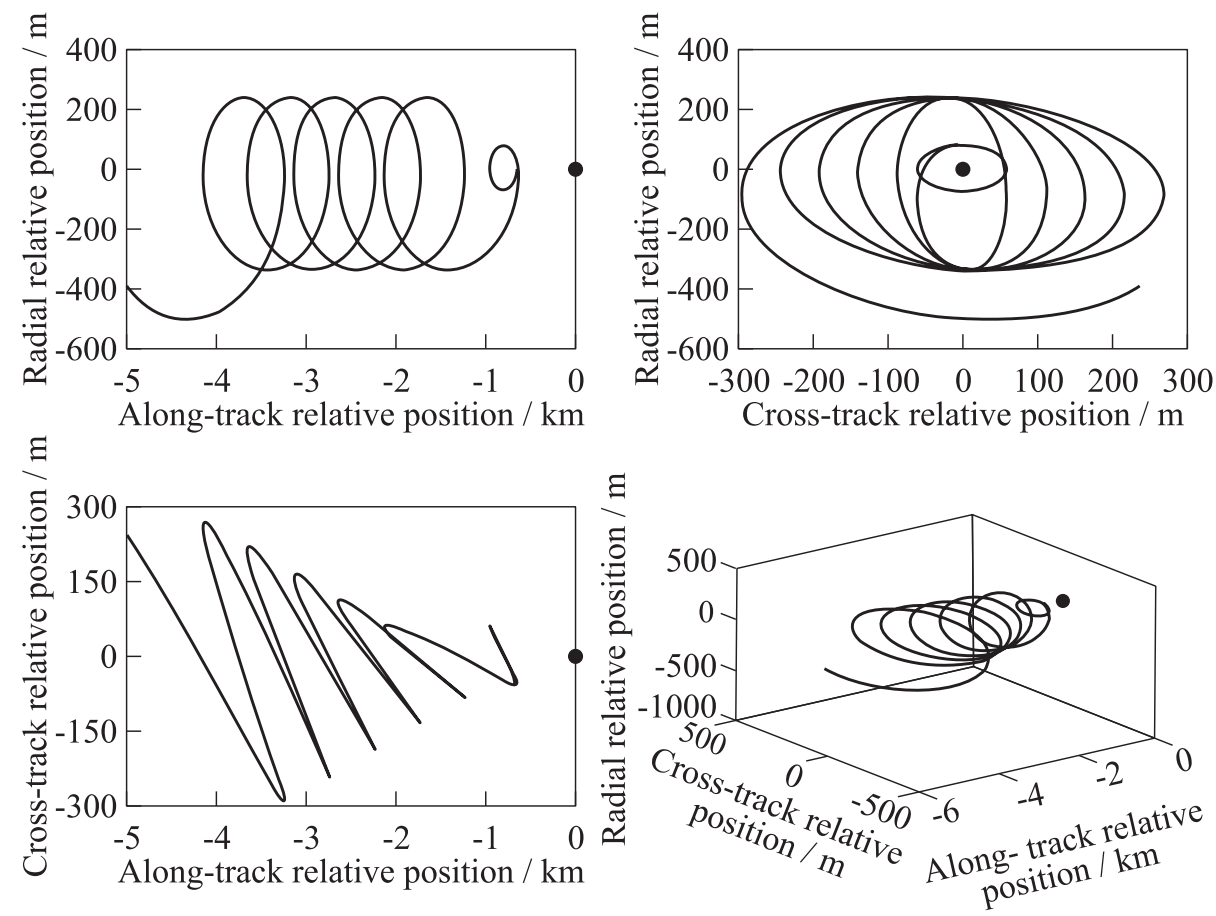

Figure 2 Radial, tangential and cross-track relative positions: approach with 4 tangential and 10-cross track manoeuvres. The black dot indicates the target position 

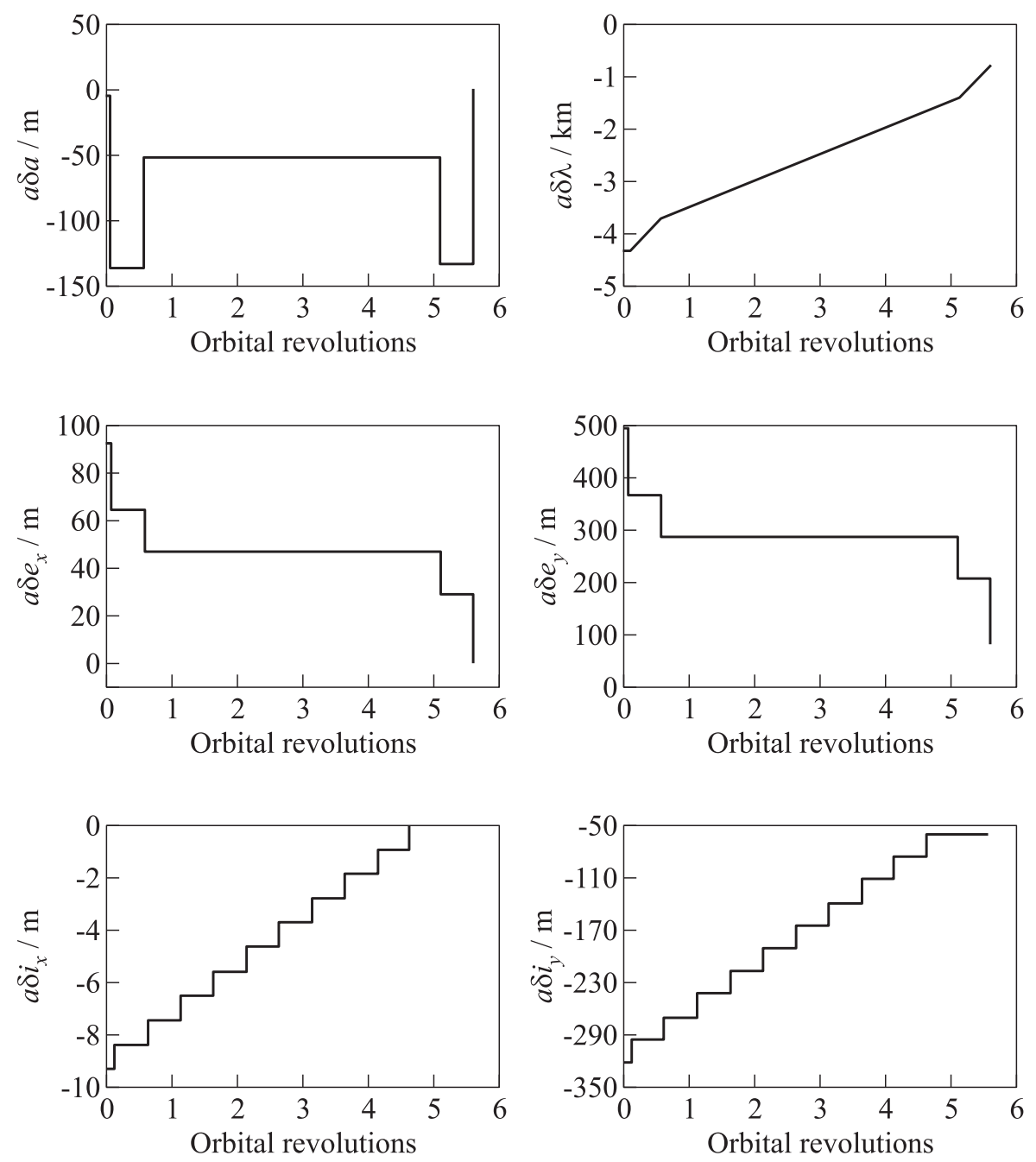

Figure 3 Relative orbital elements: approach with 4 tangential and 10 cross-track manoeuvres

Depending on the allowed time for the drift period, a small $\delta v$ along track is sufficient to introduce a drift and a second manoeuvre to terminate the drift.

The optimal propellant consumption strategy would foresee four along-track manoeuvres (two at the beginning, then a drift phase, and two at the end) and no radial manoeuvres. Four manoeuvres are generally necessary; otherwise, Eq. (3) is overestimated. The optimality is guaranteed due to the fact that changes of 
the ROE can generally be achieved with less tangential $\delta v$ than the radial $\delta v$ (see Eq. (3)).

A typical trajectory for ideal conditions (no navigation errors, no uncertainties) is plotted in Figs. 2 and. 3. The drift before the 2nd and after the 3rd manoeuvre is quite high which is disadvantageous in a failure case. Between these two manoeuvres, the radial component of the relative orbit $a \delta e$ is constant.

On the other hand, the line-of-sight based navigation requires manoeuvres for full observability (see section 4). Therefore, the strategy with two along-track manoeuvres at the beginning and two at the end is not the preferred solution from the navigation point of view. A reasonable compromise between propellant consumption and navigation requirements is the aforementioned spiral approach with approximately 10 radial manoeuvres. The number of radial manoeuvres depends on the duration foreseen for the approach. Two radial manoeuvres are

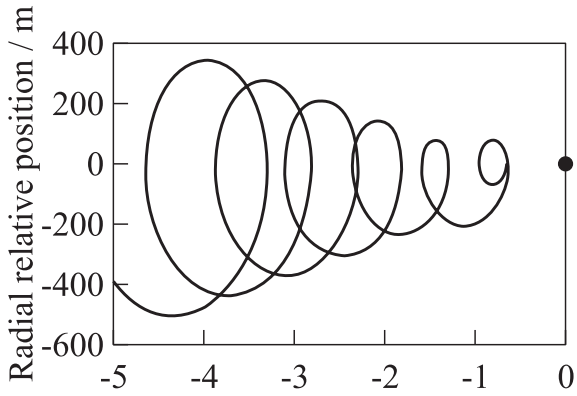

Along-track relative position / $\mathrm{km}$

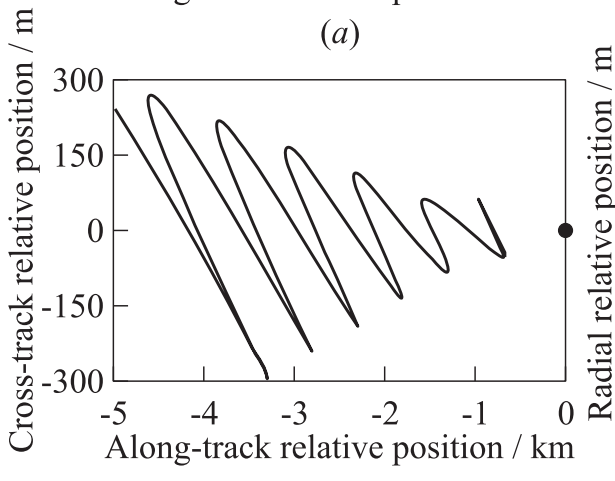

(c)

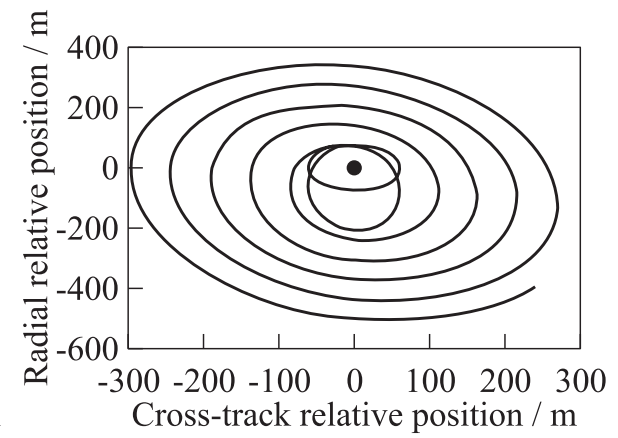

(b)

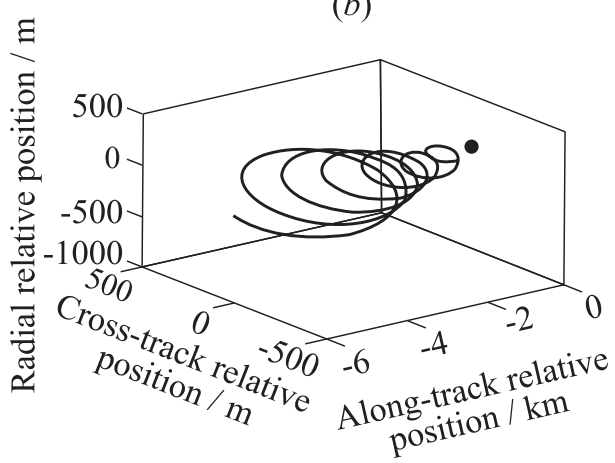

(d)

Figure 4 Radial, tangential, and cross-track relative positions: spiral approach with 10 radial, 2 tangential and 10 cross-track manoeuvres. The black dot indicates the target position 

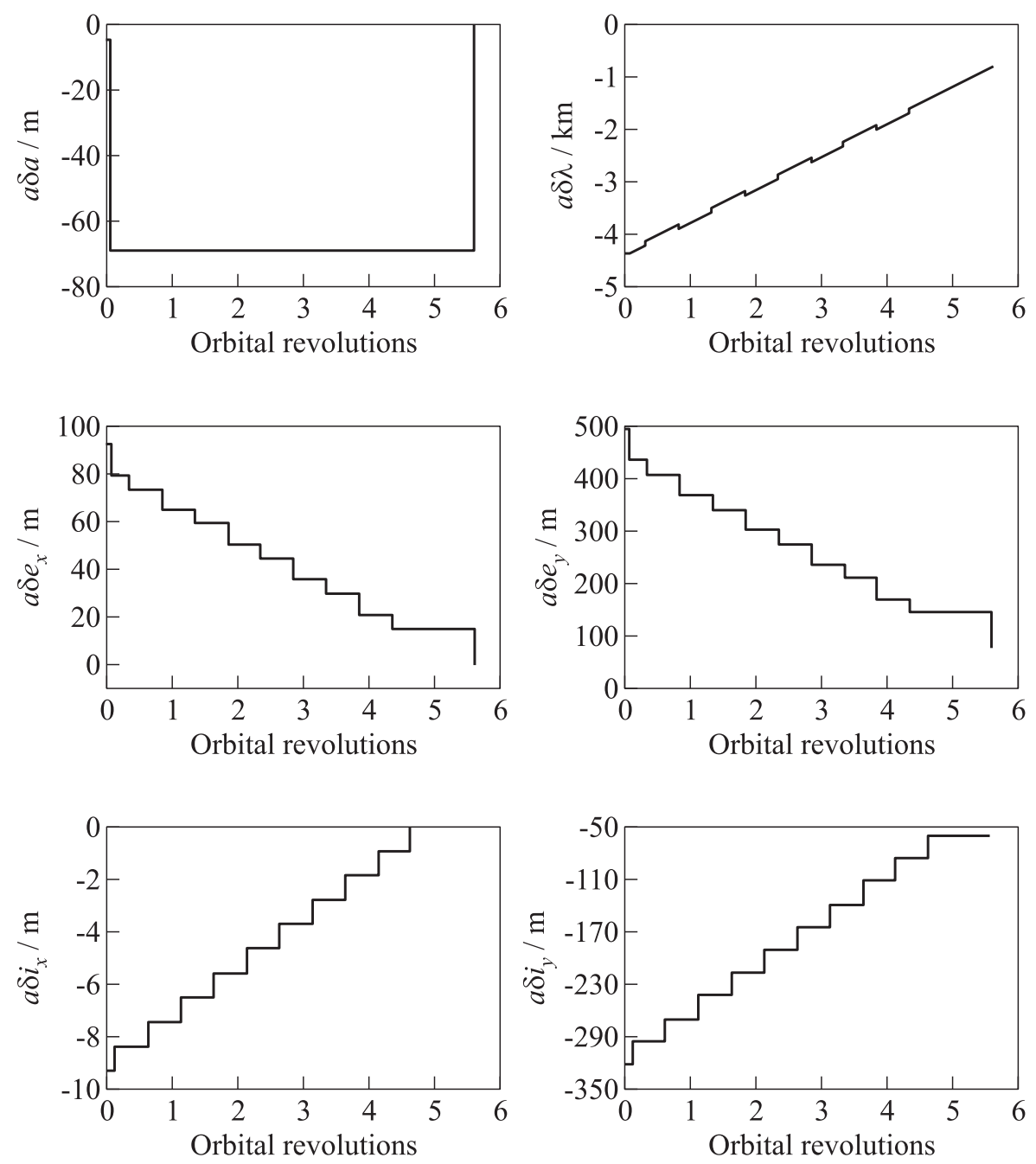

Figure 5 Relative orbital elements: Spiral approach with 10 radial, 2 tangential and 10 cross-track manoeuvres

executed per orbital period. Here, 5 orbital periods are allowed for the spiral approach. The rationale behind this choice is driven by operational aspects: the spiral approach should fit into one work day at the ground station.

The trajectory is plotted in Figs. 4 and 5. The spiral approach is clearly visible in the lower right plot in Fig. $4 d$. Figure 5 shows the corresponding successive reductions in $a \delta e$ and $a \delta i$. 
Table 1 Propellant consumption for the two approach strategies

\begin{tabular}{lcccc}
\hline Manoeuvre & $\delta v_{R}$ & $\delta v_{T}$ & $\delta v_{N}$ & $\delta v$ \\
\hline R0, T4, N10 & 0 & 0.2383 & 0.2905 & 0.5288 \\
R10, T2, N10 & 0.3289 & 0.0738 & 0.2905 & 0.6932 \\
\hline
\end{tabular}

The consumption for these two approach strategies is provided in Table 1. The total propellant consumption for the spiral approach is about $23 \%$ higher than for the strategy with 4 along-track manoeuvres. Note that $\delta v_{N}$ for the cross-track component is identical for the two approaches. When looking at the radial and along-track components only, the difference is more than $40 \%$. However, the absolute difference is only $0.1645 \mathrm{~m} / \mathrm{s}$. When comparing to the required propellant for the phasing prior to the relative navigation phase, this difference can be neglected.

During the spiral approach, two radial and two cross-track manoeuvres are commanded per orbital revolution, approximately equally distributed, which is optimal from the navigation point of view regarding the observability.

\section{RELATIVE NAVIGATION FILTER}

The relative navigation is based on line-of-sight measurements using a far range camera. The lack of observability is due to the fact that geometrically similar relative orbits lead to the same measurements. For example, a smaller relative orbit at a closer distance will lead to the same measurements as a larger relative orbit at a larger distance (Fig. 6). If manoeuvres are performed which are modifying the relative orbit, the change of the relative orbit depends on the size and direction of the manoeuvre. This known expected change of the relative orbit leads to full observability. The combination of line-of-sight measurements before and after the manoeuvre eliminates the ambiguity as shown in Fig. 6.

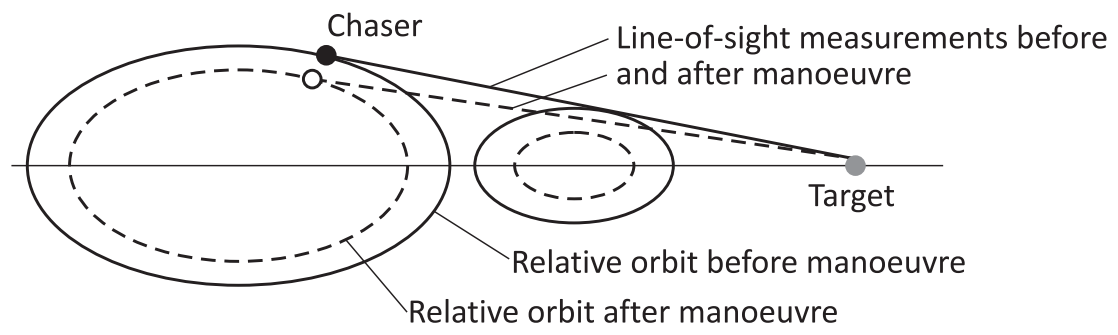

Figure 6 Observability of ROE 
An EKF has been designed to estimate the ROE. Inputs to the Kalman filter are the commanded forces, the current attitude, and the line-of-sight measurements.

The predicted state estimate $a \delta \vec{\alpha}(t)$ is:

$$
a \delta \vec{\alpha}(t+d t)=\Phi \cdot a \delta \vec{\alpha}(t)
$$

where the state transition matrix $\Phi$ including the $J_{2}$-term is

$$
\Phi=\left[\begin{array}{cccccc}
1 & 0 & 0 & 0 & 0 & 0 \\
-1.5 n_{\omega} d t & 1 & 0 & 0 & -10.5 \gamma \sin (2 i) n_{\omega} d t & 0 \\
0 & 0 & 1 & -\dot{\varphi} d t & 0 & 0 \\
0 & 0 & \dot{\varphi} d t & 1 & 0 & 0 \\
0 & 0 & 0 & 0 & 1 & 0 \\
0 & 0 & 0 & 0 & 3 \gamma \sin ^{2}(i) n_{\omega} d t & 1
\end{array}\right]
$$

Here,

$$
\gamma=0.5 J_{2} \frac{R_{\mathrm{eq}}^{2}}{a^{2}} ; \quad \dot{\varphi}=1.5 \gamma n_{\omega}\left(5 \cos ^{2} i-1\right)
$$

where $R_{\text {eq }}$ denotes the equatorial radius of the Earth. The differential drag is taken into account by

$$
\begin{aligned}
& a \delta a(t+d t)=a \delta a(t)-d_{D} \\
& a \delta \lambda(t+d t)=a \delta \lambda(t)+0.75 d_{D} n_{\omega} d t
\end{aligned}
$$

with

$$
d_{D}=\Delta B \rho a^{2} n_{\omega} d t
$$

$\Delta B$ being the difference between the chaser and target ballistic coefficients ( $B=C_{D} A / m$ where $C_{D}$ is the aerodynamic drag coefficient; $A$ is the crosssection area; and $m$ the mass of chaser and target, respectively) and $\rho$ being the atmospheric density.

Thruster forces in radial, along-track, and cross-track directions $F_{R}, F_{T}$, and $F_{N}$ during the time step $d t$ are taken into account by

$$
a \delta \delta \vec{\alpha}_{F_{\mathrm{RTN}}}=\frac{d t}{m_{C} n_{\omega}}\left(\begin{array}{c}
2 F_{T} \\
-2 F_{R} \\
F_{R} \sin u+2 F_{T} \cos u \\
-F_{R} \cos u+2 F_{T} \sin u \\
F_{N} \cos u \\
F_{N} \sin u
\end{array}\right)
$$

where $m_{C}$ is the chaser mass. The predicted estimate covariance matrix $P(t)$ is

$$
P(t+d t)=\Phi P(t) \Phi^{\mathrm{T}}+Q
$$


where $Q$ is the covariance matrix of the process noise. The measurement update requires the estimation of the predicted measurements. The line-of-sight (LoS) unit vector towards the target satellite is predicted from the current estimated relative position. Only the components in radial and cross-track directions are relevant for the EKF. The predicted LoS measurement is approximated by

$$
\operatorname{LoS}_{\text {predicted }}=\vec{z}_{1}=\left(\begin{array}{c}
-\frac{r_{N}}{r_{T}} \\
-\frac{r_{R}}{r_{T}}
\end{array}\right) .
$$

The derivative of the measurement vector $\vec{z}_{1}$ with regard to the position in the radial, tangential, and cross-track (RTN) directions is:

$$
\frac{\partial \vec{z}_{1}}{\partial r_{\mathrm{RTN}}}=\left[\begin{array}{ccc}
0 & \frac{r_{N}}{r_{T}^{2}} & -\frac{1}{r_{T}} \\
-\frac{1}{r_{T}} & \frac{r_{R}}{r_{T}^{2}} & 0
\end{array}\right] .
$$

The derivative of $\vec{z}_{1}$ with regard to the velocity is zero. The derivative of radial, tangential, and cross-track positions and velocity in the RTN frame with respect to the ROE:

$$
\frac{\partial\left(\begin{array}{l}
r_{\mathrm{RTN}} \\
v_{\mathrm{RTN}}
\end{array}\right)}{\partial \mathrm{ROE}}=\left[\begin{array}{cccccc}
1 & 0 & -\cos u & -\sin u & 0 & 0 \\
0 & 1 & 2 \sin u & -2 \cos u & 0 & 0 \\
0 & 0 & 0 & 0 & \sin u & -\cos u \\
0 & 0 & n_{\omega} \sin u & -n_{\omega} \cos u & 0 & 0 \\
-1.5 n_{\omega} & 0 & 2 n_{\omega} \cos u & 2 n_{\omega} \sin u & 0 & 0 \\
0 & 0 & 0 & 0 & n_{\omega} \cos u & n_{\omega} \sin u
\end{array}\right]
$$

Therefore, the observation model is:

$$
\begin{aligned}
& H=\left[\frac{\partial z_{1}}{\partial r_{\mathrm{RTN}}} \frac{\partial z_{1}}{\partial v_{\mathrm{RTN}}}\right] \frac{\partial\left(\begin{array}{l}
r_{\mathrm{RTN}} \\
v_{\mathrm{RTN}}
\end{array}\right)}{\partial \mathrm{ROE}}
\end{aligned}
$$

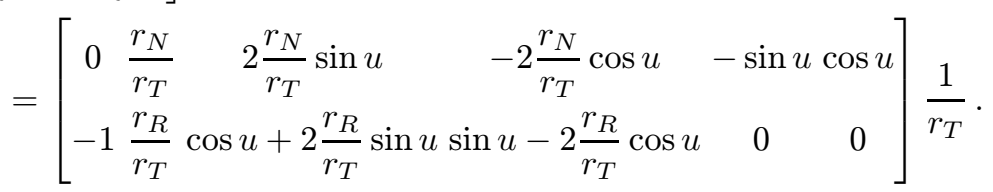

The optimal Kalman gain is

$$
K=P H^{\mathrm{T}}\left(H P H^{\mathrm{T}}+R\right)^{-1}
$$


where $R$ is the covariance matrix of the measurement noise. The updated state estimate and estimate covariance are:

$$
\begin{aligned}
a \delta \vec{\alpha} & =a \delta \vec{\alpha}_{\text {prev }}+K\left(z-z_{1}\right) \\
P & =P_{\text {prev }}-K H P_{\text {prev }}
\end{aligned}
$$

\section{SIMULATION RESULTS}

In this section, the Monte-Carlo simulation results are presented. The uncertainties and disturbances include the initial conditions, thrust level and direction, measurement noise, mass, differential drag and gravitational terms up to 20th degree and order. The line-of-sight measurements are provided by a camera model taking into account eclipse phases and several other disturbances like illumination conditions of the target, camera resolution, focal length, and sensor noise. During eclipse phases, no measurements are available. Outside eclipse, the illuminated area of the target determines the measured LoS. The commanded forces also differ from the realised forces by the thrust level and direction. The main uncertainties are listed below:

$$
\begin{aligned}
& \text { thrust level }-5 \% \text {; } \\
& \text { thrust direction }-1^{\circ} \text {; } \\
& \text { chaser mass }-5 \% \text {; } \\
& \text { target mass }-5 \% \text {; } \\
& \text { drag coefficient chaser }-30 \% \text {; } \\
& \text { drag coefficient target }-30 \% \text {; } \\
& \text { initial ROE - 30\%; and } \\
& \text { LoS measurement - camera model. }
\end{aligned}
$$

Figure 7 shows the navigation error on the ROE for 100 simulations. The grey lines are the navigation errors, and the black lines are 3 times the square root of the corresponding entries in the covariance matrix $P$. The filter converges quickly after the first manoeuvres. The only exception is the semimajor axis difference $a \delta a$. Here, the initial guess is as accurate as the results of the ROE filter. The reason behind this is that the semimajor axis can be determined quite accurately from ground because it depends directly on the orbital period. The along-track position of the relative orbit $a \delta \lambda$ converges more slowly than the other ROE. The error is about one order of magnitude larger than the others. This result is not surprising. The along-track position is expected to be the 

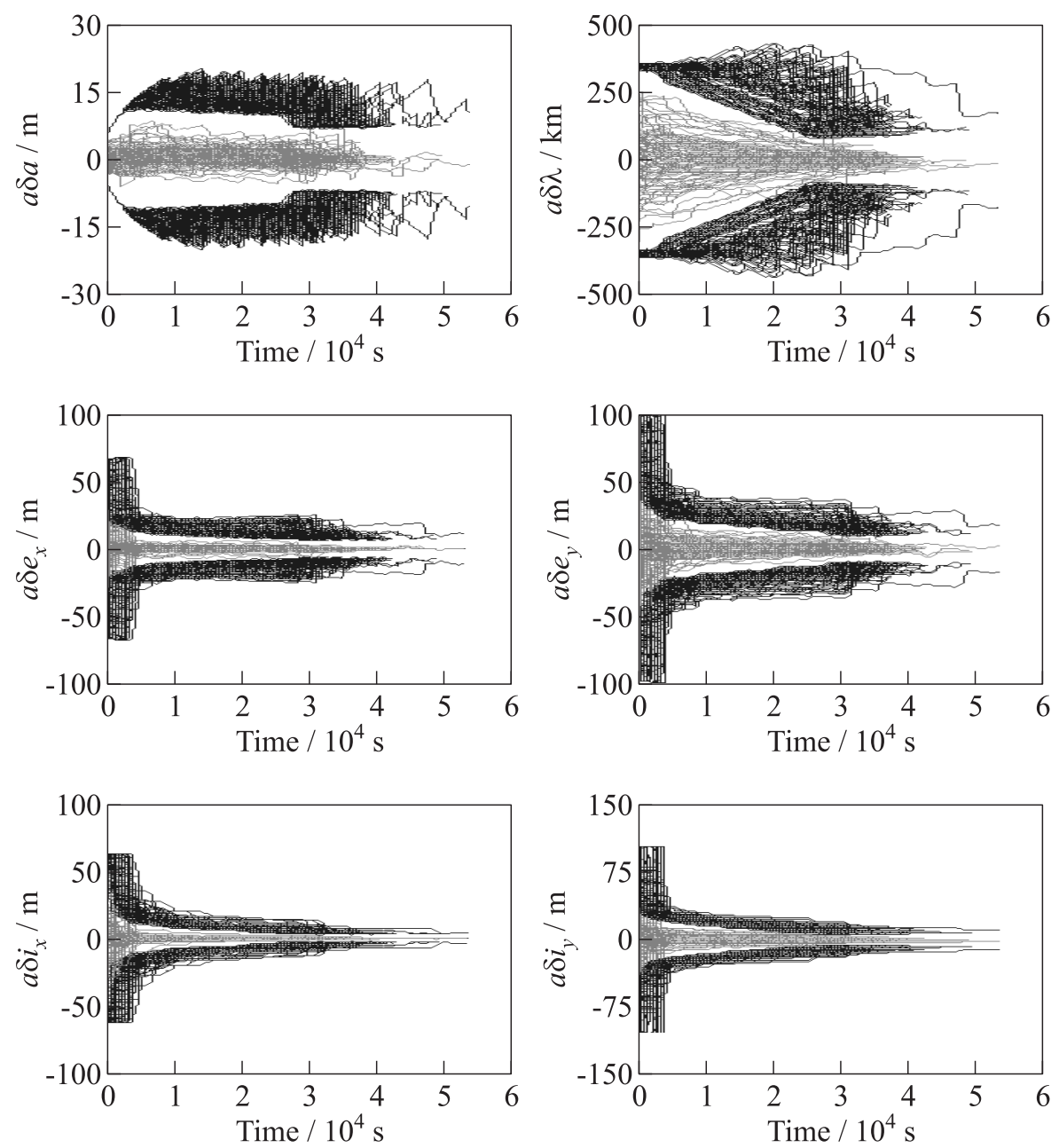

Figure 7 The ROE navigation filter performance

one with the largest uncertainties. That is exactly the reason for the guidance strategy based on $e / i$-vector separation.

The temporal evolution of the ROE is plotted in Fig. 8. The safe corridor is clearly visible in the $a \delta e_{y}$ and $a \delta i_{y}$ plots. The corridor is also visible in Fig. $9 b$ showing the relative position in the RTN frame. Note that some simulation runs are longer than 5 orbital periods. In these cases, the nominal first tangential manoeuvre according to Eq. (5) would lead to a violation of the safety corridor for the prescribed duration of 5 orbital periods. 

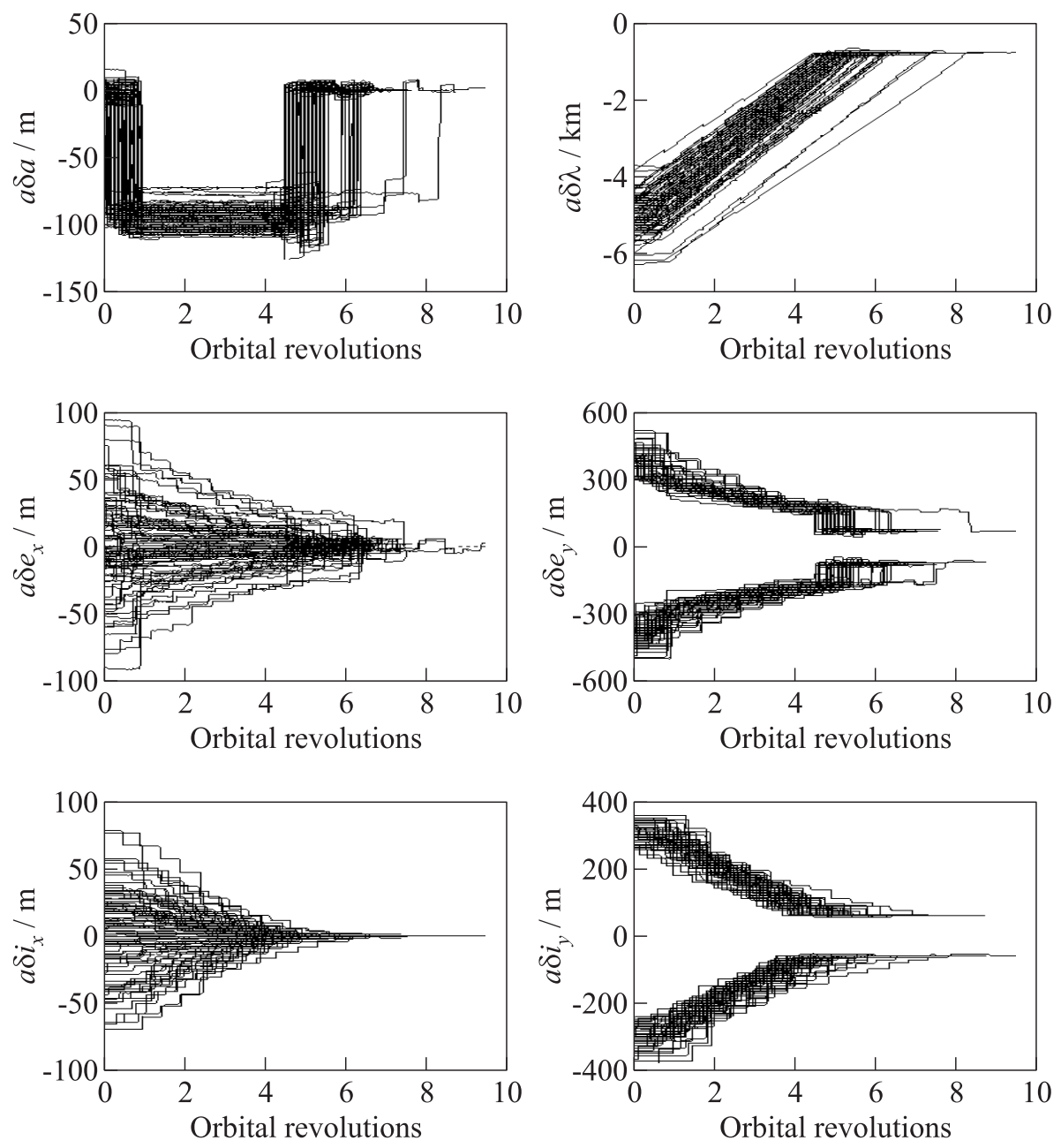

Figure 8 Relative orbital elements

This potential violation is detected by prediction of the ROE based on Eq. (1) and - if necessary - corrected by increasing the duration of the spiral approach accordingly. This correction decreases the propellant consumption due to the increased drift duration.

Figure 10 shows the propellant consumption in terms of $\delta v$ for the three directions: radial, along-track, and cross-track (RTN). The cases with very low propellant consumption correspond to the cases with increased duration. 


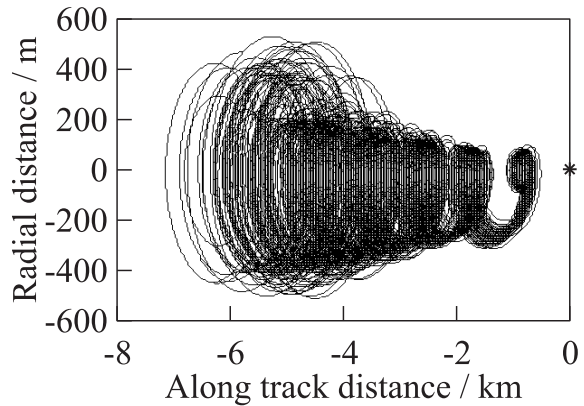

(a)

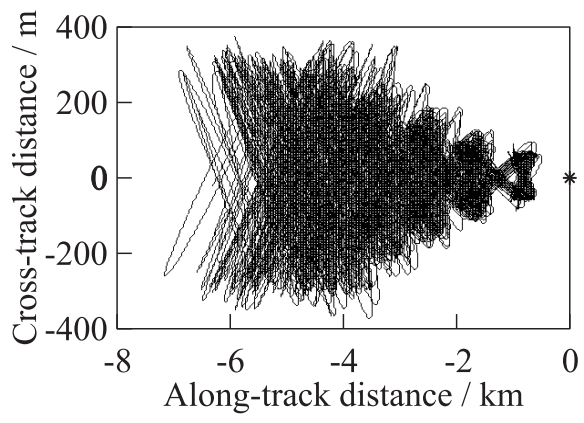

(c)

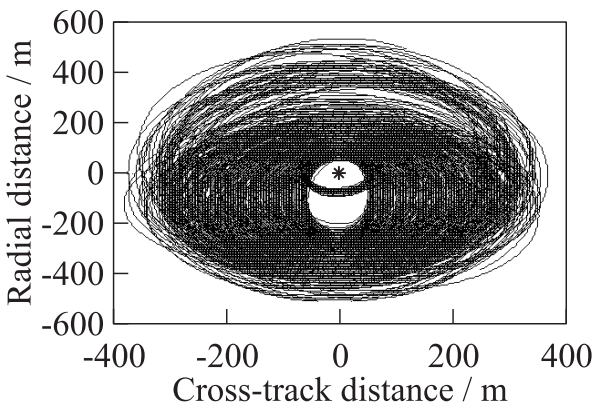

(b)

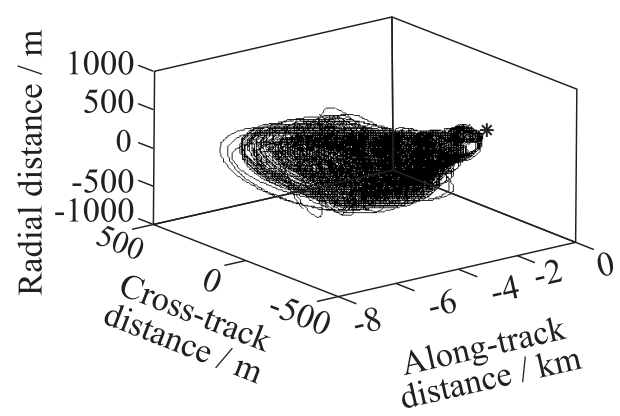

(d)

Figure 9 Relative position in the RTN

\section{SUMMARY AND CONCLUDING REMARKS}

This paper presents a guidance strategy based on a spiral approach with $e / i$ vector separation. This guidance strategy guaranties a passive safe approach to the uncooperative target while the propellant consumption is close to the theoretical minimum. This approach strategy also ensures enough manoeuvres for observability of the ROE with line-of-sight measurements. The navigation employs a classical EKF. The performance of the guidance and navigation approach has been demonstrated in Monte-Carlo simulations.

\section{REFERENCES}

1. Clohessy, W.H., and R.S. Wiltshire. 1960. Terminal guidance system for satellite rendezvous. J. Aerosp. Sci. 27(9):653-658. 

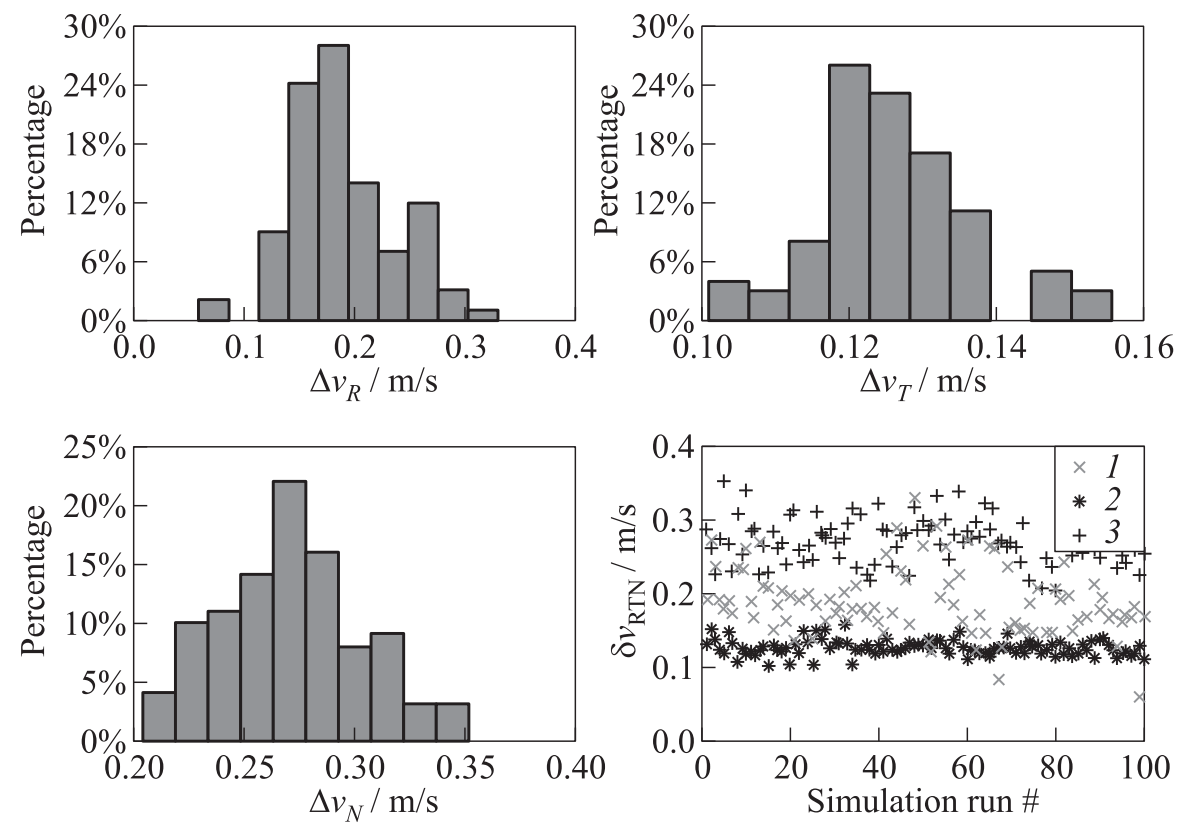

Figure 10 Consumption: $\delta v_{\mathrm{RTN}}: 1-\mathrm{R} ; 2-\mathrm{T} ; 3-\mathrm{N}$

2. Kahle, R., B. Schlepp, and F. Meissner. 2011. TerraSAR-X / TanDEM-X formation acquisition - analysis and flight results. 21st AAS/AIAA Space Flight Mechanics Meeting. New Orleans, LA.

3. D'Amico, S., E. Gill, and O. Montenbruck. 2006. Relative orbit control design for the prisma formation flying mission. AIAA Guidance, Navigation and Control Conference and Exhibit. Keystone, CO.

4. Stastny, N. B., R. A. Bettinger, and F. R. Chavez. 2008. Comparison of the extended and unscented Kalman filters for angle based relative navigation. AIAA/AAS Astrodynamics Specialist Conference and Exhibit. Honolulu, HI.

5. Posch, A., A. O. Schwientek, J. Sommer, and W. Fichter. 2012. Comparison of filter techniques for relative state estimation of in-orbit servicing missions. IFAC Workshop on Embedded Guidance, Navigation and Control in Aerospace. Bangalore, India.

6. D'Amico, S. 2010. Autonomous formation flying in low Earth orbit. Technical University of Delft. PhD Thesis. 